\title{
On Perturbation of Non-Linear Equations in Banach Spaces
}

By

\author{
Yoshikazu Kobayashi* and Kazuo Kobayasi**
}

\section{Introduction}

Let $A$ be a dissipative operator in a Banach space $X$ and let $X_{0}$ be a subset of $X$. In this paper we study the "range" condition

$$
R(I-\lambda A) \supset X_{0} \quad \text { for } \quad \lambda>0 \text {. }
$$

Condition (1) states that given $f \in X_{0}$ and $\lambda>0$ there is a $u \in D(A)$ satisfying the equation $u-\lambda A u \ni f$. It is also known that under condition (1) (with $X_{0}=\overline{D(A)}$ ) $A$ generates a contraction semigroup on $\overline{D(A)}$ (cf. [5, Theorem I]).

Our first purpose is to discuss sufficient conditions for (1). In general, the direct verification of (1) is not easy. We shall give some conditions on $A$ which implies condition (1). Our conditions seem to be weaker than (1) and hence would be easy to check. We note, however, that our conditions are, in fact, equivalent to (1).

Next, given dissipative operators $A$ and $B$, we consider the perturbation problem of Kato type; in which one wants to show

$$
R(I-\lambda(A+B)) \supset X_{0} \quad \text { for } \quad \lambda>0
$$

if $B$ is small relative to $A$ in a certain sense. Our second purpose is to give conditions on $A$ and $B$ under which $A+B$ satisfies our conditions mentioned above.

From the same point of view, in case $X^{*}$ is uniformly convex, Kato

Communicated by S. Matsuura, June 2, 1975. Revised June 14, 1976.

* Faculty of Engineering, Niigata University, Nagaoka, 940, Japan.

** Department of Mathematics, Waseda University, Tokyo, 160, Japan. 
[8] and Brezis-Pazy [3] gave sufficient conditions for (1), e.g., for every $x \in D(A)$ there exist a neighborhood $U_{x}$ of $x$ and a sequence $\varepsilon_{n} \downarrow 0$ such that

$$
R\left(I-\varepsilon_{n} A\right) \supset X_{0} \cap U_{x}
$$

(which implies Kato's local m-dissipativeness condition); and Kato treated the perturbation problem (2).

In Section 2, we discuss conditions equivalent to condition (1). We shall treat this problem in the setting where $X$ is a general Banach space and further relax the conditions imposed by Kato and BrezisPazy. Our method is based on a generation theorem essentially due to Takahashi [12]: If for every $x \in D_{a}(A)$

$$
\operatorname{dist}(R(I-\lambda A), x)=o\left(\lambda^{2}\right) \quad \text { as } \quad \lambda \downarrow 0
$$

holds, then $A$ generates a contraction semigroup on $\overline{D(A)}$. In Section 3 we shall treat the perturbation problem (2) and give some perturbation theorems of Kato type in the setting where $X$ is a general Banach space.

\section{Preliminaries}

Throughout this paper $X$ denotes a real Banach space with the dual space $X^{*}$ and the bidual space $X^{* *}$. The norms in these spaces are denoted by \|\| and the natural pairing between $x \in X$ and $f \in X^{*}$ is denoted by $\langle x, f\rangle$. We write by $F$ the duality mapping of $X$ into $X^{*}$, that is, $F(x)=\left\{f \in X^{*} ;\left\langle x, f>=\|x\|^{2}=\|f\|^{2}\right\}\right.$ for $x \in X$. We set

$$
<y, x\rangle_{s}=\sup \{<y, f>; f \in F(x)\}
$$

and

$$
<y, x\rangle_{i}=\inf \{<y, f>; f \in F(x)\}, \quad x, y \in X
$$

For the properties of $\langle\cdot, \cdot\rangle_{s}$ and $\langle\cdot, \cdot\rangle_{i}$ we refer to [5; Lemma 2.16]. In particular, we note that the function $\left.<\cdot, \cdot\rangle_{s}(\text { resp. }<\cdot, \cdot\rangle_{i}\right)$ from $X \times X$ into $R^{1}$ is upper (resp. lower) semi-continuous with respect to the strong topology of $X \times X$. 
We mean by an operator $A$ in $X$ a subset of $X \times X$, and set $A x=$ $\{y ;[x, y] \in A\}, D(A)=\{x ; A x \neq \phi\}$ and $R(A)=\cup_{x \in D(A)} A . x$. The sets $D(A)$ and $R(A)$ are called the domain and range of $A$ respectively. An operator $A$ in $X$ is said to be dissipative if

$$
<y_{1}-y_{2}, x_{1}-x_{2}>_{i} \leqq 0
$$

for each $\left[x_{j}, y_{j}\right] \in A, j=1,2$. If, in place of (1.1), $\left\langle y_{1}-y_{2}, x_{1}-x_{2}\right\rangle_{s}$ $\leqq 0$ holds, then we say that $A$ is dissipative in the sense of Browder. It is well known that $A$ is dissipative if and only if the resolvent $J_{\lambda}=$ $(I-\lambda A)^{-1}$ of $A$ is single-valued and a contraction for all $\lambda>0$, where I denotes the identity on $X$.

Let $A$ be an operator in $X$. For each $x \in D(A)$ we set

$$
\|A x\|=\inf \{\|y\| ; y \in A x\} .
$$

Following Takahashi [13], we introduce the set $D_{a}(A)$ which consists of all $x \in X$ such that there exists a sequence $\left\{x_{n}\right\}$ in $D(A)$ satisfying that $\lim _{n \rightarrow \infty} x_{n}=x$ and $\left\|A x_{n}\right\|$ is bounded. Also we define

$$
|A x|= \begin{cases}\inf \{M ; & \left.x_{n} \in D(A), \lim _{n \rightarrow \infty} x_{n}=x, \varlimsup_{n \rightarrow \infty}\left\|A x_{n}\right\| \leqq M\right\} \\ \infty & \text { if } \quad x \notin D_{a}(A)\end{cases}
$$

As is easily seen from the definitions of $D_{a}(A)$ and $|\cdot|$, we have:

(a) $|A x| \leqq\|A x\|$ for $x \in D(A)$ and $D(A) \subset D_{a}(A) \subset \overline{D(A)}$.

(b) The function $|A x|$ from $X$ into $[0, \infty]$ is lower semi-continuous with respect to the strong topology of $X$.

(c) If $A$ is a dissipative operator and satisfies that $R(I-\lambda A) \supset \overline{D(A)}$ for all sufficiently small $\lambda>0$, then $x \in D_{a}(A)$ if and only if $\left\|A_{\lambda} x\right\|$ is bounded as $\lambda \downarrow 0$, where $A_{\lambda}=\lambda^{-1}\left(J_{\lambda}-I\right)$. In this case we have $|A x|=$ $\lim _{\lambda \downarrow 0}\left\|A_{\lambda} x\right\|$.

Remark 1.1. Under the assumptions of (c) stated above we see that $D_{a}(A)$ equals to the generalized domain $\hat{D}(A)$ which was introduced by Crandall [4]. When $X$ is a reflexive Banach space, $D_{a}(A)=D(A)$ if and only if $A$ is almost demi-closed, that is, if $\left[x_{n}, y_{n}\right] \in A, x_{n} \rightarrow x$ and $y_{n} \rightarrow y$, then $x \in D(A)$. (We denote by " $\rightarrow$ " and " $\rightarrow$ " strong con- 
vergence and weak convergence respectively.) Also see [13] for further properties of $D_{a}(A)$.

Let $X_{0}$ be a subset of $X$. A one-parameter family $\{T(t) ; t \geqq 0\}$ of single-valued operators from $X_{0}$ into itself is called a contraction semigroup on $X_{0}$ if it satisfies

(i) $T(0) x=x$ for $x \in X_{0}, T(s+t)=T(s) T(t)$ for $s, t \geqq 0$,

(ii) $\|T(t) x-T(t) y\| \leqq\|x-y\|$ for $x, y \in X_{0}$ and $t \geqq 0$,

(iii) $\lim _{t \downarrow 0} T(t) x=x$ for $x \in X_{0}$.

Finally, we state a generation theorem of semigroups which is a slight generalization of Theorem III in [12]. The proof of this theorem will be given in the Appendix.

Theorem. Let $A$ be a dissipative operator in $X$. Suppose:

(R) For each $x \in D_{a}(A)$ and each $M>0$ there are a neighborhood $U$ of $x$ and a positive constant $K$ with the property that for every $u$ $\in D_{a}(A) \cap U$ with $|A u| \leqq M$ there exists a positive sequence $\left\{\varepsilon_{n}\right\}$ such that $\varepsilon_{n} \rightarrow 0$ and

$$
\operatorname{dist}\left(R\left(I-\varepsilon_{n} A\right), u\right) \leqq K \varepsilon_{n}^{2} \quad \text { for all } n \geqq 1 .
$$

Then there exists a unique contraction semigroup $\{T(t) ; t \geqq 0\}$ on $\overline{D(A)}$ such that for each $x \in \overline{D(A)}, u(t)=T(t) x$ satisfies

$$
\|u(t)-u\|^{2}-\|u(s)-u\|^{2} \leqq 2 \int_{s}^{t}<v, u(\eta)-u>_{s} d \eta
$$

for all $[u, v] \in A$ and all $s, t \in[0, \infty)$ with $s \leqq t$. Moreover, if $x \in D_{a}(A)$, then $u(t) \in D_{a}(A)$ and

$$
|A u(t)|=\lim _{h \downarrow 0} h^{-1}\|u(t+h)-u(t)\|
$$

for all $t \geqq 0$. 


\section{Range conditions}

In this section we introduce some range conditions for an operator in $X$ and investigate the relationship among them.

Let $C$ be a subset of $X$. We say that an operator $A$ in $X$ satisfies condition $\left(R_{1}\right)$ (resp. $\left(R_{2}\right)$ ) on $C$ if for each $x \in D_{a}(A)$, each $w \in C$ and each $M>0$ there are a neighborhood $U$ of $x$ and a positive constant $K$ satisfying the following $(*)$ (resp. $(* *))$ :

(*) For each $u \in D_{a}(A) \cap U$ with $|A u| \leqq M$, there are a neighborhood $V$ of $u$ and a positive sequence $\left\{\delta_{n}\right\}$ such that $\delta_{n} \rightarrow 0$ and

$$
\operatorname{dist}\left(R\left(I-\delta_{n} A\right), v\right) \leqq K \delta_{n}^{2}
$$

for all $n$ and all $v \in \operatorname{seg}[u, v] \cap V$.

(**) For each $u \in D_{a}(A) \cap U$ with $|A u| \leqq M$ and each $v \in C$, there is a positive sequence $\left\{\delta_{n}\right\}$ such that $\delta_{n} \rightarrow 0$ and

$$
\operatorname{dist}\left(R\left(I-\delta_{n}(A-v), u\right) \leqq K \delta_{n}^{2} \quad \text { for all } n .\right.
$$

Here $\operatorname{seg}[u, w]$ denotes the segment from $u$ to $w$. We also introduce the following condition which is stronger than $\left(R_{2}\right)$ :

$\left(R_{3}\right)$ For each $x \in D_{a}(A)$ and each $w \in X$ there is a positive sequence $\left\{\delta_{n}\right\}$ such that $\delta_{n} \rightarrow 0$ and

$$
R\left(I-\delta_{n}(A-w)\right) \ni x \quad \text { for all } n .
$$

Theorem 2.1. Let $A$ be a closed, dissipative operator in $X$ and $C$ be a convex subset of $X$ including $D_{a}(A)$. Then the following (i) and (ii) are equivalent:

(i) $R(I-\lambda A) \supset C$ for all $\lambda>0$.

(ii) A satisfies condition $\left(R_{1}\right)$ on $C$.

In addition, if $C$ is a linear subspace, then (i) and (ii) are equivalent to the following:

(iii) A satisfies condition $\left(R_{2}\right)$ on $C$.

Corollary 2.2. Let $A$ be a dissipative operator in $X$. Then the following three conditions are equivalent: 
(i) $A$ is m-dissipative, that is, $A$ is dissipative and $R(I-\lambda A)=X$ for all $\lambda>0$.

(ii) A satisfies condition $\left(R_{3}\right)$.

(iii) $A$ is closed and satisfies condition $\left(R_{1}\right)$ or $\left(R_{2}\right)$ on $X$.

Remark 2.3. Theorem 2.1 and Corollary 2.2 extend some results in Kato [8] and Brezis-Pazy [3] to the case of general Banach spaces.

To prove the theorem and the corollary we prepare the following

Lemma 2.4. Let $C$ be a subset of $X$ including $D_{a}(A), w$ be an element of $C$ and $\lambda$ be a positive number. Put $B=\lambda A-I+w$. Assume that $A$ satisfies $\left(R_{1}\right)$ on $C$ or that $C$ is a linear subspace and $A$ satisfies $\left(R_{2}\right)$ on $C$. Then $B$ satisfies condition $(R)$.

Proof. We first note that $D_{a}(A)=D_{a}(B)$. Let $x$ be an element of $D_{a}(B)$ and $M^{\prime}$ be a positive number. Set $M=\left(M^{\prime}+1+\|x-w\|\right) / \lambda$. Then, for these $x$ and $M$ there exist a positive number $K$ and an open ball $U=U(x, r)$ with the center $x$ and rudius $r \leqq 1$ such that $(*)$ (resp. $(* *))$ holds. Let $u$ bc any element of $D_{a}(B) \cap U$ such that $|B u| \leqq M^{\prime}$. Then, clcarly, $|A u| \leqq\left(M^{\prime}+r+\|x-w\|\right) / \lambda \leqq M$, so that there is a sequence $\left\{\delta_{n}\right\}$ satisfying the properties in $(*)$ (resp. $(* *)$ ) with a neighborhood $V$ of $u$.

First, assume that $(*)$ holds. Then, setting $\varepsilon_{n}=\delta_{n} /\left(\lambda-\delta_{n}\right)$ and $v_{n}$ $=\left(u+\delta_{n}, w\right) /\left(1+\varepsilon_{n}\right)$ and noting that $v_{n} \in \operatorname{seg}[u, w] \cap \mathrm{V}$ for all sufficiently large $n$, we have that $\operatorname{dist}\left(R\left(I-\delta_{n} A\right), v_{n}\right) \leqq K \delta_{n}^{2}$ and hence

$$
\begin{aligned}
& \operatorname{dist}\left(R\left(I-\varepsilon_{n} B\right), u\right) \\
& \leqq\left(1+\varepsilon_{n}\right) \operatorname{dist}\left(R\left(I-\delta_{n} A\right), v_{n}\right) \leqq \lambda^{2} K \varepsilon_{n}^{2}
\end{aligned}
$$

for all sufficiently large $n$.

Next, assume that $(* *)$ holds and $C$ is a linear subspace. Setting $v=\lambda^{-1}(u-w)$ and noting that $v \in C$, we get $\operatorname{dist}\left(R\left(I-\delta_{n}(A-v)\right), u\right) \leqq K \delta_{n}^{2}$ for all $n$. Hence,

$$
\begin{aligned}
& \operatorname{dist}\left(R\left(I-\varepsilon_{n} B\right), u\right) \\
& \leqq\left(1+\varepsilon_{n}\right) \operatorname{dist}\left(R\left(I-\delta_{n}(A-v)\right), u\right) \leqq \lambda^{2} K \varepsilon_{n}^{2} .
\end{aligned}
$$

Thus we see that $B$ satisfies condition $(R)$.

Q.E.D. 
Proof of Theorem 2.1. Let $w \in C$ be an arbitrary element and $\lambda$ be an arbitrary positive number. Put $B=\lambda A-I+w$. We first assume (ii) in the theorem. By the Theorem in the Section 1 and Lemma 2.4, there exists a contraction semigroup $\{T(t) ; t \geqq 0\}$ on $\overline{D(B)}$ such that

$$
|B T(t) x|=\lim _{h \downarrow 0} h^{-1}\|T(t+h) x-T(t) x\|
$$

for $t \geqq 0$ and $x \in D_{a}(B)$. Since $B+I$ is dissipative, it can be proved that $\|T(t) x-T(t) y\| \leqq e^{-t}\|x-y\|$ for $t \geqq 0$ and $x, y \in \overline{D(B)}$ (for example, see [10]). This fact and (2.1) give that

$$
\begin{aligned}
\|T(t+h) x-T(t) x\| & \leqq e^{-t}\|T(h) x-x\| \\
& <e^{-t} \lim _{n \rightarrow \infty} \sum_{h=1}^{n} e^{(k-1) h / n}\|T(h / n) x-x\| \\
& =e^{-t}\left(1-e^{-h}\right)|B x|
\end{aligned}
$$

for all $t, h \geqq 0$. This shows that $z=\lim _{t \rightarrow \infty} T(t) x$ exists for $x \in D_{a}(B)$ and $|B z|=0$. For $|B z| \leqq \lim _{t \rightarrow \infty}|B T(t) x| \leqq \lim _{t \rightarrow \infty}\left(\lim _{h \rightarrow 0}^{t \rightarrow \infty} e^{-t} h^{-1}\left(1-e^{-h}\right)|B x|\right)=0$. Here we have used property (b) in the Section 1 . The fact that $|B z|=0$ implies that therc is a sequence $\left\{\left[x_{n}, y_{n}\right]\right\}$ in $B$ such that $x_{n} \rightarrow z$ and $y_{n} \rightarrow 0$. Since $B$ is closed as well as $A$, we have $[z, 0] \in B$, that is, $w \in R(I-\lambda A)$. Thus we have proved that (ii) implies (i). Similarly, we can prove that (iii) implies (i). Conversely, it is evident to see that (i) implies (ii) and (iii).

Q.E.D.

Proof of Corollary 2.2. It suffices to show that if $A$ satisfies $\left(R_{3}\right)$, then $A$ is closed. Let $\left\{\left[x_{k}, y_{k}\right]\right\}$ be a sequence in $A$ such that $x_{k} \rightarrow x$ and $y_{k} \rightarrow y$. Since $|A x| \leqq \lim _{k \rightarrow \infty}\left|A x_{k}\right| \leqq \lim _{k \rightarrow \infty}\left\|y_{k}\right\|<\infty$, it follows from $\left(R_{3}\right)$ that there exists an $\varepsilon=\varepsilon(x, y)>0$ such that $R(I-\varepsilon(A-y)) \ni x$, that is, $x_{0}-x=\varepsilon\left(y_{0}-y^{\prime}\right)$ for some $\left[x_{0}, y_{0}\right] \in A$. Letting $k$ tend to $\infty$ in $<y_{0}$ $\left.-y_{k}, x_{0}-x_{k}\right\rangle_{i} \leqq 0$, we have $\left\langle y_{0}-y, x_{0}-x\right\rangle_{i} \leqq 0$ and hence

$$
\begin{aligned}
\left\|x-x_{0}\right\|^{2} & =<x_{0}-x, x_{0}-x>_{i} \\
& \leqq \varepsilon<y_{0}-y, x_{0}-x>_{i} \leqq 0 .
\end{aligned}
$$

Thus $x=x_{0}$ and $y=y_{0}$. Hence $A$ is closed.

Q.E.D. 


\section{Perturbation Problems}

In this section, let $A$ be an operator in $X$ and let $B$ be a singlevalued operator in $X$. We say that $B$ is locally $A$-bounded with $A$ bound $<1$ if $D(A) \subset D(B)$ and for each $x \in \overline{D(A)}$ there are a neighborhood $U$ of $x$ and constants $K \geqq 0, L \geqq 0$ with $L<1$ such that

$$
\|B u\| \leqq K+L\|A u\| \quad \text { for any } \quad u \in D(A) \cap U .
$$

We consider the following type of local Lipschitz conditions:

(L.1) $D(A) \subset D(B)$, and for each $x \in D_{a}(A)$ and each $M>0$ there are a neighborhood $U$ of $x$ and a constant $K \geqq 0$ such that

$$
\|B u-B v\| \leqq K\|u-v\|
$$

whenver $u, v \in D(A) \cap U,\|A u\| \leqq M$ and $\|A v\| \leqq M$.

(L.2) $D(A) \subset D(B)$, and for each $x \in D_{a}(A)$ there are a neighborhood $U$ of $x$ and nonnegative constants $K$ and $L<1$ such that

$$
\|B u-B v\| \leqq K\|u-v\|+L\|A u-A v\| \| \text { for any } u, v \in D(A) \cap U .
$$

Remark 3.1. If there exist nondecreasing functions $k_{i}(t)$ on $[0, \infty)$, $i=1,2$, such that

$$
\|B u-B v\| \leqq\left(k_{1}(\|A u\|)+k_{2}(\|A v\|)\right)\|u-v\| \quad \text { for all } u, v \in D(A),
$$

then $B$ satisfies local Lipschitz condition (L.1). This type of condition has been discussed by Kato [7].

Our main result of this section is as follows.

Theorem 3.2. Assume that $A$ is m-dissipative and that $B$ is dissipative, locally A-bounded with A-bound $<1$ and satisfies local Lipschitz condition (L.1) or (L.2). If at least one of $A$ and $B$ is dissipative in the sense of Browder, then $A+B$ is $m$-dissipative and $D_{a}(A+B)=D_{a}(A)$.

Remark 3.3. If $X$ is reflexive in Theorem 3.2, then $A$ is almost demi-closed (see Kenmochi [9] and Remark 1.1). Therefore Theorem 3.2 is an extension of Theorem 11.1 in [8] to the case of general Banach 
spaces.

Lemma 3.4. If $B$ is locally A-bounded with $A$-bound $<1$, then $D_{a}(A+B)=D_{a}(A)$.

Proof. Let $x \in D_{a}(A)$. Then there exist a sequence $\left\{x_{n}\right\} \subset D(A)$ and a constant $M$ such that $x_{n} \rightarrow x$ and $\left\|A x_{n}\right\| \leqq M$. Let $U$ be a neighborhood of $x$ satisfying (3.1). Since we may assume that $x_{n} \in U$ for all $n$, we have $\left\|B x_{n}\right\| \leqq K+L M$, and hence $\left\|(A+B) x_{n}\right\| \leqq\left\|A x_{n}\right\|+\left\|B x_{n}\right\| \leqq$ $K+(L+1) M$. This shows $x \in D_{a}(A+B)$. Conversely, let $x \in D_{a}(A+B)$. Then there exist a sequence $\left\{x_{n}\right\} \subset D(A)$ and a constant $M$ such that $x_{n} \rightarrow x$ and $\left\|(A+B) x_{n}\right\| \leqq M$. Also, by (3.1), we have $\left\|A x_{n}\right\| \leqq\left\|(A+B) x_{n}\right\|$ $+\left\|B x_{n}\right\| \leqq M+K+L\left\|A x_{n}\right\|$. Since $L<1$, we obtain $\left\|A x_{n}\right\| \leqq(1-L)^{-1}(M$ $+K)$, so that $x \in D_{a}(A)$.

Q.E.D.

The local Lipschitz condition (L.1) or (L.2) on $B$ implies a local range condition of $A+B$ on $D_{a}(A)$. Precisely we have the following lemmas.

Lemma 3.5. Suppose that $A$ is m-dissipative and $D_{a}(A+B)=D_{a}(A)$. If $B$ satisfies local Lipschitz condition (L.1), then $A+B$ satisfies condition $\left(R_{3}\right)$.

Lemma 3.6. Suppose that $A$ is m-dissipative and $D_{a}(A+B)=D_{a}(A)$. If $B$ satisfies local Lipschitz condition (L.2) with $L<1 / 2$, then $A+B$ satisfies condition $\left(R_{3}\right)$.

Proof of Lemma 3.5. Let $a \in D_{a}(A+B)=D_{a}(A)$ and $w \in X$. We want to show that $a \in R(I-\varepsilon(A+B-w))$ for all sufficiently small $\varepsilon>0$. We may assume that $w=0$ since (3.2) is true even if $B$ is replaced by $B-w$. Hence we shall show that there is a $\delta>0$ such that the equation

$$
z-\varepsilon(A+B) z \ni a
$$

has a solution $z \in D(A)$ if $0<\varepsilon<\delta$. Put $J_{\lambda}=(I-\lambda A)^{-1}$ for $\lambda>0$. We note that $J_{\lambda}: X \rightarrow D(A)$ is a contraction. Then (3.4) is equivalent to

$$
z=I_{\varepsilon}(a+\varepsilon B z) \text {. }
$$


Let $D\left(B_{1}\right)=\left\{x \in X ; \lim _{\lambda \downarrow 0} B J_{\lambda} x\right.$ exists $\}$ and define an operator $B_{1}$ with domain $D\left(B_{1}\right)$ by

$$
B_{1} x=\lim _{\lambda \downarrow 0} B J_{\lambda} x \quad \text { for } \quad x \in D\left(B_{1}\right) .
$$

If $x \in D_{a}(A)$, then $J_{\lambda} x \in D(A)$ and $J_{\lambda} x \rightarrow x$ as $\lambda \downarrow 0$ with $\left\|A J_{\lambda} x\right\| \leqq\left\|A_{\lambda} x\right\|$ $\leqq|A x|$. Hence we see by (3.2) that $D_{a}(A) \subset D\left(B_{1}\right)$ and $B_{1} x=B x$ if $x \in$ $D(A)$. Therefore, (3.4) is also equivalent to the equation

$$
z=J_{\varepsilon}\left(a+\varepsilon B_{1} z\right)
$$

Thus it suffices to show that there is a $\delta>0$ such that (3.7) has a solution if $0<\varepsilon<\delta$. To this end we use the fixed point theorem. Let $M>|A a|+2\left\|B_{1} a\right\|$. By the condition (L.1), for these $a$ and $M$ there exist positive numbers $r$ and $K$ such that (3.2) holds for $u, v \in D(A) \cap B_{2 r}$ with $\|A u\| \leqq M$ and $\|A v\| \leqq M$, where $B_{r}=B(a, r)$ and $B(a, r)$ denotes a closed ball with center $a$ and rudius $r$. If $u, v \in D_{a}(A) \cap B_{r}$ and $|A u|$ $\leqq M,|A v| \leqq M$, then $J_{\lambda} u, J_{\lambda} v \in D(A) \cap B_{2 r},\left\|A J_{\lambda} u\right\||\leqq| A u \mid \leqq M$ and $\left\|A J_{\lambda} v\right\|$ $\leqq|A v| \leqq M$ for all sufficiently small $\lambda>0$, and hence

$$
\left\|B J_{\lambda} u-B J_{\lambda} v\right\| \leqq K\left\|J_{\lambda} u-J_{\lambda} v\right\| \leqq K\|u-v\|
$$

for all sufficiently small $\lambda>0$. Letting $\lambda \downarrow 0$, we have

$$
\left\|B_{1} u-B_{1} v\right\| \leqq K\|u-v\|
$$

for $u, v \in D_{a}(A) \cap B_{r}$ such that $|A u| \leqq M$ and $|A v| \leqq M$. Put $\rho=\min \{r$, $\left.\left(M-|A a|-2\left\|B_{1} a\right\|\right) / 2 K\right\}$ and

$$
\Sigma=\left\{x ; x \in D_{a}(A) \cap B_{\rho} \text { and }|A x| \leqq M\right\} .
$$

Obviously, $a \in \Sigma$ and $\Sigma$ is closed in $X$ by virtue of the lower semicontinuity of $|A x|$ in $x$. Choose a $\delta>0$ so that $\delta<\rho /(K \rho+|A a|+$ $\left.\left\|B_{1} a\right\|\right)$ and let $\varepsilon$ be an arbitrary number in $(0, \delta)$. Now let us define an operator $G$ by

$$
G x=J_{\varepsilon}\left(a+\varepsilon B_{1} x\right) \quad \text { for } \quad x \in \Sigma
$$

with $D(G)=\Sigma$. In order to show that $G$ has a fixed point, we observe from (3.8) that for any $x \in \Sigma$ 


$$
\begin{aligned}
\left\|B_{1} x\right\| & \leqq\left\|B_{1} x-B_{1} a\right\|+\left\|B_{1} a\right\| \leqq K\|x-a\|+\left\|B_{1} a\right\| \\
& \leqq K \rho+\left\|B_{1} a\right\|
\end{aligned}
$$

and hence

$$
\begin{aligned}
\|G x-a\| & \leqq\left\|J_{\varepsilon}\left(a+\varepsilon B_{1} x\right)-J_{\varepsilon} a\right\|+\left\|J_{\varepsilon} a-a\right\| \\
& \leqq \varepsilon\left(\left\|B_{1} x\right\|+|A a|\right) \leqq \varepsilon\left(K \rho+\left\|B_{1} a\right\|+|A a|\right) \leqq \rho
\end{aligned}
$$

Furthermore, noting that $\|A G x\| \leqq\left\|A_{\varepsilon}\left(a+\varepsilon B_{1} x\right)\right\|=\varepsilon^{-1} \| a+\varepsilon B_{1} x-J_{\varepsilon}(a+$ $\left.\varepsilon B_{1} x\right)\|\leqq\| B_{1} x\left\|+\varepsilon^{-1}\right\| a-G x \|$ for any $x \in \Sigma$, we have by (3.9) and (3.10) that

$$
|A G x| \leqq\left\|A G x\left|\|\leqq 2 K \rho+2\| B_{1} a \|+\right| A a \mid \leqq M\right.
$$

for any $x \in \Sigma$. Hence $G$ maps $\Sigma$ into itself. Also, $G$ is a strict contraction; in fact, we obtain from (3.8) that

$$
\|G x-G y\| \leqq \varepsilon\left\|B_{1} x-B_{1} y^{\prime}\right\| \leqq K \varepsilon\left\|x-y^{\prime}\right\| \leqq K \delta\|x-y\|
$$

for any $x, y \in \Sigma$. Hence $G$ has a fixed point $z \in \Sigma$, that is, $z=G z=$ $J_{\varepsilon}\left(a+\varepsilon B_{1} z\right)$.

Q.E.D.

Proof of Lemma 3.6. Just as in the proof of Lemma 3.5, it suffices to show that if $a \in D_{a}(A)$, then (3.4) has a solution $z$ in $D_{a}(A)$ for all sufficiently small $\varepsilon>0$. Let $a \in D_{a}(A)$. Note that (3.4) is equivalent to (3.5). If we set $y=a+\varepsilon B z$ in (3.5), then we have $z=J_{\varepsilon} y$ and hence

$$
y=a+\varepsilon B J_{\varepsilon} y .
$$

Conversely, if $y$ is a solution of (3.11), then clearly $z=J_{\varepsilon} y$ satisfies (3.5). Therefore we shall show that for sufficiently small $\varepsilon>0$, (3.11) has a solution $y$. We use again the fixed point theorem. By assumption there are positive constants $r, K$ and $L$ with $L<1 / 2$ such that (3.3) holds true for any $u, v \in D(A) \cap B_{2 r}$. In this case we note that (3.1) holds true with $U=B_{2 r}$ and $K$ replaced by a suitable $K^{\prime} \geqq 0$. Take $\sigma$ with $2 L<\sigma$ $<1$, and set $\delta=\min \left\{r(1-\sigma) /\left(K^{\prime}+|A a|\right),(\sigma-2 L) / K\right\}$ and $\Sigma=B_{r}$. Let $\varepsilon \in(0, \delta)$ be arbitrarily fixed. We then define an operator $G$ with $D(G)$ $=\Sigma$ by $G x=a+\varepsilon B J_{\varepsilon} x$. If $x \in \Sigma$, then 


$$
\begin{aligned}
\left\|J_{\varepsilon} x-a\right\| & \leqq\left\|J_{\varepsilon} x-J_{\varepsilon} a\right\|+\left\|J_{\varepsilon} a-a\right\| \leqq\|x-a\|+\left\|J_{\varepsilon} a-a\right\| \\
& \leqq\|x-a\|+\varepsilon|A a| \leqq 2 r,
\end{aligned}
$$

that is, $J_{\varepsilon} x \in B_{2 r}$, because $\varepsilon|A a| \leqq r(1-\sigma) \leqq r$. Since $J_{\varepsilon} x, J_{\varepsilon} y \in D(A) \cap B_{2 r}$ for any $x, y \in \Sigma$, we have by (3.3)

$$
\begin{aligned}
\|G x-G y\| & \leqq \varepsilon\left\|B J_{\varepsilon} x-B J_{\varepsilon} y\right\| \\
& \leqq \varepsilon K\left\|J_{\varepsilon} x-J_{\varepsilon} y\right\|+\varepsilon L\left\|A J_{\varepsilon} x-A J_{\varepsilon} y\right\| \\
& \leqq(\varepsilon K+2 L)\|x-y\| \leqq(\delta K+2 L)\|x-y\| \leqq \sigma\|x-y\|
\end{aligned}
$$

for any $x, y \in \Sigma$. This shows that $G$ is a strict contraction. Furthermore $G$ maps $\Sigma$ into itself. In fact, if $x \in \Sigma$, then

$$
\|G x-a\| \leqq\|G x-G a\|+\|G a-a\| \leqq \sigma\|x-a\|+\varepsilon\left\|B J_{\varepsilon} a\right\| .
$$

Since $J_{\varepsilon} a \in B_{2 r},(3.1)$ with $K$ replaced by $K^{\prime}$ implies that $\left\|B J_{\varepsilon} a\right\| \leqq$ $K^{\prime}+L\left\|A J_{c} a\right\| \leqq K^{\prime}+|A a|$. Hence

$$
\|G x-a\| \leqq \sigma r+\varepsilon\left(K^{\prime}+|A a|\right) \leqq r
$$

for $x \in \Sigma$, that is, $G x \in B_{r}=\Sigma$ for $x \in \Sigma$ by the definition of $\delta$. Hence $G$ has a fixed point $y \in \Sigma$, so that $y=G y=a+\varepsilon B J_{\varepsilon} y$.

Q.E.D.

Proof of Theorem 3.2. At first, assume that $B$ satisfies local Lipschitz condition (L.1). By Lemmas 3.4 and 3.5, $A+B$ satisfies condition $\left(R_{3}\right)$. Since $A+B$ is dissipative, the assertion follows from Corollary 2.2. Next, assume that $B$ satisfies local Lipschitz condition (L.2). If we can take $L<1 / 2$ in (3.3), then Lemmas 3.4 and 3.6 imply that $A+B$ satisfies condition $\left(R_{3}\right)$, so that the assertion follows from Corollary 2.2 again. We shall now use the continuity argument due to Kato [8] to remove this restriction (see the proof of Theorem 11.1 in [8]). Consider the family of operators $A+t B, 0 \leqq t \leqq 1$. We note that each $A+t B$ is dissipative and $D_{a}(A+t B)=D_{a}(A)$ by Lemma 3.4 since $t B$ is locally $A$-bounded with $A$-bound $<1$. Thus Lemma 3.6 assures that $A+t B$ is $m$-dissipative if $0 \leqq t \leqq 1 / 2$. On the other hand, (3.3) implies

$$
\|B u-B v\| \leqq K\|u-v\|+L(\|(A+t B) u-(A+t B) v\|+t\|B u-B v\|),
$$

and hence 


$$
\|B u-B v\| \leqq(1-t L)^{-1}(K\|u-v\|+L\|(A+t B) u-(A+t B) v\|)
$$

for $u, v \in D(A) \cap U$. If $A+t B$ is known to be $m$-dissipative, then by the above result $A+t^{\prime} B=(A+t B)+\left(t^{\prime}-t\right) B$ is $m$-dissipative whenever ( $t^{\prime}-$ $t)(1-L t)^{-1} \leqq 1 / 2$. Therefore, $A+t B$ is $m$-dissipative for all $t<1$. Furthermore, since $(1-t)(1-L t)^{-1} \leqq 1 / 2$ for all $t$ sufficiently near 1 , we see that $A+B$ is $m$-dissipative.

Q.E.D.

\section{Appendix}

We here give a proof of the Theorem in Section 1 .

Lemma A1. Let $A$ be a dissipative operator in $X$ and let $u$ : $[0, T] \rightarrow X$ be a continuous function such that (1.2) holds for all $[u, v]$ $\in A$ and $s, t \in[0, T]$ with $s \leqq t$. Suppose that

$$
\frac{\lim }{h \downarrow 0} h^{-1} \operatorname{dist}(R(I-h A), u(t))=0 \quad \text { for } \quad t \in[0, T) .
$$

Then for $t \in[0, T), u(t) \in D_{a}(A)$ if and only if $h^{-1}\|u(t+h)-u(t)\|$ is bounded as $h \downarrow 0$. In this case, (1.3) holds and $\|u(r)-u(t)\| \leqq|A u(t)|$ $|r-t|$ for any $r \in[t, T]$.

Proof. We follow the argument of Bénilan [2] and Takahashi [12]. Let $t$ be a real number in $[0, T)$. First, suppose that $u(t) \in D_{a}(A)$. We can choose a sequence $\left\{\left[u_{n}, v_{n}\right]\right\} \subset A$ such that $u_{n} \rightarrow u(t)$ and $\left\|v_{n}\right\| \leqq$ $|A u(t)|+1 / n$. It now follows from (1.2) that

$$
\left\|u(r)-u_{n}\right\|^{2}-\left\|u(t)-u_{n}\right\|^{2} \leqq 2(|A u(t)|+1 / n) \int_{t}^{r}\left\|u(\eta)-u_{n}\right\| d \eta
$$

for all $r \in[t, T]$ and all $n \geqq 1$. Letting $n$ tend to $\infty$, we obtain that

$$
\|u(r)-u(t)\|^{2} \leqq 2|A u(t)| \int_{t}^{r}\|u(\eta)-u(t)\| d \eta
$$

which implies

$$
\|u(r)-u(t)\| \leqq|A u(t)|(r-t)
$$

for all $r \in[t, T]$, and hence 


$$
\varlimsup_{h \downarrow 0} h^{-1}\|u(t+h)-u(t)\| \leqq|A u(t)| .
$$

We next assume that $h^{-1}\|u(t+h)-u(t)\|$ is bounded as $h \downarrow 0$. Then a generalized sequence $\left\{h^{-1}(u(t+h)-u(t)) ; 0<h<T-t\right\}$ in $X^{* *}$ has a cluster point $z \in X^{* *}$ with respect to the weak*-topology of $X^{* *}$ and $z$ satisfies $\|z\| \leqq \lim _{h \downarrow 0} h^{-1}\|u(t+h)-u(t)\|$. By the assumption (4.1) there exist sequences $\left\{\delta_{n}\right\}$ and $\left\{\left[x_{n}, y_{n}\right]\right\} \subset A$ such that $\delta_{n} \rightarrow 0$ and $x_{n}-\delta_{n} y_{n}-$ $u(t)=o\left(\delta_{n}\right)$. Since

$$
\begin{aligned}
<h^{-1}(u(t+h)-u(t)), f> & \leqq(2 h)^{-1}\left(\left\|u(t+h)-x_{n}\right\|^{2}-\left\|u(t)-x_{n}\right\|^{2}\right) \\
& \leqq h^{-1} \int_{t}^{t+h}<y_{n}, u(\eta)-x_{n}>_{s} d \eta
\end{aligned}
$$

for all $f \in F\left(u(t)-x_{n}\right)$, we obtain that $-\|z\|\left\|u(t)-x_{n}\right\| \leqq<z, f>\leqq<y_{n}$, $u(t)-x_{n}>_{s^{*}}$ Hence

$$
\begin{aligned}
\left\|u(t)-x_{n}\right\|^{2} & =<u(t)-x_{n}, u(t)-x_{n}>_{i} \\
& \leqq-\delta_{n}<y_{n}, u(t)-x_{n}>_{s}+\left\|o\left(\delta_{n}\right)\right\|\left\|u(t)-x_{n}\right\| \\
& \leqq \delta_{n}\|z\|\left\|u(t)-x_{n}\right\|+\left\|o\left(\delta_{n}\right)\right\|\left\|u(t)-x_{n}\right\|
\end{aligned}
$$

which gives

$$
\left\|y_{n}\right\| \leqq \delta_{n}^{-1}\left(\left\|u(t)-x_{n}\right\|+\left\|o\left(\delta_{n}\right)\right\|\right) \leqq\|z\|+\delta_{n}^{-1}\left\|o\left(\delta_{n}\right)\right\| .
$$

This implies that $u(t) \in D_{a}(A)$ and $|A u(t)| \leqq\|z\| \leqq \lim _{h \downarrow 0} h^{-1}\|u(t+h)-u(t)\|$.

Q.E.D.

Lemma A2. Under the assumptions of the Theorem, for each $x \in$ $D_{a}(A)$ there exist a positive number $T_{x}$ and a unique continuous function $u:\left[0, T_{x}\right] \rightarrow X$ such that $u(0)=x$ and (1.2) holds for any $[u, v] \in A$ and $s, t \in\left[0, T_{x}\right]$ with $s \leqq t$. Moreover, $|A u(t)|$ is nonincreasing in $t$.

Proof. Let $x$ be an element of $D_{a}(A)$ and set $M=2|A x|$. By condition $(R)$ we can take positive constants $r=r(x)$ and $K=K(x)$ such that for any $\varepsilon>0$ and any $u \in D_{a}(A) \cap B(x, r)$ with $|A u| \leqq M$ there is $a \delta>0$ satisfying $\delta \leqq \varepsilon$ and

$$
\operatorname{dist}(R(I-\delta A), u) \leqq K \delta^{2} / 3
$$


Setting $T_{x}=\min \{1,2 r /(M+4 K), M / 4 K\}, t_{0}^{n}=0$ and $x_{0}^{n}=x$, we define $t_{h}^{n}$ and $\left[x_{h}^{n}, y_{h}^{n}\right] \in A$ for $k \geqq 1$ such that

(i) $t_{h}^{n}=t_{h-1}^{n}+h_{k}^{n}$ with $h_{k}^{n} \in\left(\mu_{k}^{n} / 2, \mu_{k}^{n}\right)$, where $\mu_{k}^{n}$ is the supremum of all $\mu$ such that $0<\mu<1 / n, t_{h-1}^{n}+\mu<T_{x}$ and $\operatorname{dist}\left(R(I-\mu A), x_{h-1}^{n}\right)$ $\leqq 2 K \mu^{2} / 3$;

(ii) $\left[x_{h}^{n}, y_{h}^{n}\right] \in A$ and $\left\|z_{h}^{n}\right\| \leqq K h_{h}^{n}$, where $z_{h}^{n}=\left(x_{k}^{n}-x_{k-1}^{n}\right) / h_{k}^{n}-y_{k}^{n}$; in fact, it is possible to construct sequences $\left\{t_{k}^{n}\right\}_{k=1}^{\infty}$ and $\left\{\left[x_{k}^{n}, y_{k}^{n}\right]\right\}_{k=1}^{\infty}$ by the following estimates:

$$
\begin{aligned}
\left|A x_{k}^{n}\right| & \leqq\left\|y_{k}^{n}\right\| \leqq\left\|z_{k}^{n}\right\|+\left\|x_{k}^{n}-x_{k-1}^{n}\right\| / h_{k}^{n} \\
& \leqq\left|A x_{k-1}^{n}\right|+2\left\|z_{k}^{n}\right\| \\
& \leqq|A x|+2 \sum_{j=1}^{k}\left\|z_{j}^{n}\right\| \\
& \leqq|A x|+2 K t_{k}^{n} \\
& \leqq|A x|+2 K T_{x} \\
& \leqq M
\end{aligned}
$$

and hence

$$
\begin{aligned}
\left\|x_{k}^{n}-x\right\| & \leqq \sum_{j=1}^{k}\left\|x_{j}^{n}-x_{j-1}^{n}\right\| \\
& \leqq \sum_{j=1}^{k}\left(\left\|z_{j}^{n}\right\|+|A x|+2 K t_{j-1}^{n}\right) h_{j}^{n} \\
& \leqq\left(|A x|+2 K T_{x}\right) t_{k}^{n}<r .
\end{aligned}
$$

Next, we suppose that $\lim _{k \rightarrow \infty} t_{k}^{n}=t<T_{x}$. Then $z=\lim _{k \rightarrow \infty} x_{k}^{n}$ exists and belongs to $D_{a}(A) \cap B(x, r)$. Moreover, $|A z| \leqq|A x|+2 K t \leqq M \quad$ by (4.4). Hence it follows from (4.3) that there exists a $\delta>0$ such that $\delta \leqq$ $\min \left\{1 / 2 n, T_{x}-t\right\}$ and $\operatorname{dist}(R(I-\delta A), z) \leqq K \delta^{2} / 3$. However, since $\mu_{k}^{n}<2 h_{k}^{n}$ $<\delta$ for all sufficiently large $k$, the definition of $\mu_{k}^{n}$ implies that $\operatorname{dist}(R(I$ $\left.-\delta A), x_{k-1}^{n}\right)>2 K \delta^{2} / 3$ for all such $k$. Letting $k \rightarrow \infty$, we get $\operatorname{dist}(R(I$ $-\delta A), z) \geqq 2 K \delta^{2} / 3$ which contradicts the inequality $\operatorname{dist}(R(I-\delta A), z)$ $\leqq K \delta^{2} / 3$. Therefore, $\lim _{k \rightarrow \infty} t_{k}^{n}=T_{x}$ must be true. Then we define step functions $u_{n}(t)$ and $f_{n}(t)$ for $n \geqq 1$ by 


$$
u_{n}(t)=\left\{\begin{array}{ll}
x & \text { if } t=0 \\
x_{k}^{n} & \text { if } t \in\left(t_{k-1}^{n}, t_{k}^{n}\right], \\
x_{N_{n}}^{n} & \text { if } t \in\left(t_{N_{n}}^{n}, T_{x}\right]
\end{array} \quad f_{n}(t)=\left\{\begin{aligned}
z_{k}^{n} & \text { if } t \in\left(t_{k-1}^{n}, t_{k}^{n}\right] \\
-y_{N_{n}}^{n} & \text { if } t \in\left(t_{N_{n}}^{n}, T_{x}\right]
\end{aligned}\right.\right.
$$

where $k=1,2, \ldots, N_{n}$ and $N_{n}$ is an integer such that $T_{x}-t_{N_{n}}^{n} \leqq 1 / n$. We can easily see that $\left\|u_{n}(t)-u_{n}(s)\right\| \leqq$ Const. $|t-s|$ for all $s, t \in\left[0, T_{x}\right]$ and $f_{n} \rightarrow 0$ in $L^{1}\left(0, T_{x} ; X\right)$ as $n \rightarrow \infty$. Hence, by virtue of $\left[12\right.$; Theorem I] $u_{n}$ converges uniformly on $\left[0, T_{x}\right]$ to a unique continuous function $u$ such that $u(0)=x$ and (1.2) holds for any $[u, v] \in A$ and $s, t \in\left[0, T_{x}\right]$ with $s \leqq t$, and such that $\|u(t+h)-u(t)\| \leqq\|u(s+h)-u(s)\|$ for $0 \leqq s \leqq t \leqq t+h \leqq T_{x}$. Since $u(t) \in D_{a}(A)$ by (4.4), condition $(R)$ assures that $u(t)$. satisfies (4.1). Therefore, (1.3) holds for every $t \in\left[0, T_{x}\right)$ by Lemma $\mathrm{A} 1$ and $|A u(t)|$ is nonincreasing.

Q.E.D.

Proof of Theorem. Let $x \in D_{a}(A)$ and let $[0, T)$ be the largest interval on which there is a unique function $u$ such that $u(0)=x,(1.2)$ holds for any $[u, v] \in A$ and $s, t \in[0, T)$ with $s \leqq t$ and $|A u(t)|$ is nonincreasing in $t$. Suppose that $T<\infty$. Since $u(t) \in D_{a}(A)$, (4.2) gives that $\| u(r)-$ $u(t) \| \leqq(r-t)|A u(t)| \leqq(r-t)|A x|$ for $0 \leqq t \leqq r<T$. This implies that $z$ $=\lim _{t \uparrow T} u(t)$ exists and belongs to $D_{a}(A)$ again, because $|A z| \leqq \frac{\lim }{s \uparrow T}|A u(s)|$ $\leqq|A u(t)| \leqq|A x|$ for any $t \in[0, T)$ by property (b) in Section 1. Hence, by Lemma $\mathrm{A} 2$ we can extend $u$ beyond $T$. Therefore we must have $T=\infty$. Consequently, we obtain the desired semigroup.

Q.E.D.

\section{Acknowledgements}

The authors wish to thank the referee for many helpful comments.

\section{References}

[1] Barbu, V., Continuous perturbations of nonlinear $m$-accretive operators in Banach spaces, Boll. Un. Mat. Ital., 6 (1972), 270-278.

[2] Bénilan, Ph., Equations d'evolution dan un espace du Banach quelconque et applications, Thése, Orsay, (1972).

[3] Brezis, H. and Pazy, A., Accretive sets and differential equations in Banach spaces, Israel J. Math., 8 (1970), 367-383.

[4] Crandall, M. G., A generalized domain for semigroup generators, Proc. Amer. 
Math. Soc., 37 (1973), 434-440.

[5] Crandall, M. G. and Liggett, T. M., Generation of semigroups of nonlinear transformations on general Banach spaces, Amer. J. Math., 93 (1971), 265-298.

[6] Crandall, M. G. and Pazy, A., Semigroups of nonlinear contractions and dissipative sets, J. Functional Analysis, 3 (1969), 376-418.

[7] Kato, T., Nonlinear evolution equations in Banach spaces, Proc. Symp. Applied Math., 17, AMS P. R. I., (1965), 50-67.

[8] - Accretive operators and nonlinear evolution equations in Banach spaces, Proc. Symp. Pure Math., 18, Part I, AMS P. R. I. (1970), 158-161.

[9] Kenmochi, N., Remarks on the m-accretiveness of nonlinear operators, Hiroshima Math. J., 3 (1973), 61-68.

[10] Kenmochi, N. and Oharu, S., Difference approximation of nonlinear evolution equations, Publ. RIMS, Kyoto Univ., 10 (1974), 147-207.

[11] Martin, R., Differential equations on closed subsets of a Banach space, Tras. Amer. Math. Soc., 179 (1973), 399-414.

[12] Takahashi, T., Convergence of difference approximation of nonlinear evolution equations and generation of semigroups, J. Math. Soc. Japan, 28 (1976), 96113.

[13] - Invariant sets for semigroups of nonlinear operators, to appear.

[14] Webb, G., Continuous nonlinear perturbations of linear accretive operators in Banach spaces, J. Functional Analysis, 10 (1972), 191-203. 
